\section{Staphylococcus aureus Decolonization of Mice With Monoclonal Antibody Neutralizing Protein A}

\author{
Xinhai Chen, ${ }^{1,2,3}$ Yan Sun, ${ }^{1}$ Dominique Missiakas, ${ }^{1}$ and Olaf Schneewind ${ }^{1}$ \\ 'Department of Microbiology, University of Chicago, Illinois; and 'Shenzhen \\ International Institute for Biomedical Research, and ${ }^{3}$ Immunartis LLC, Shenzhen, \\ Guangdong, China
}

(See the Editorial Commentary by Kobayashi and DeLeo on pages 848-50.)

Staphylococcus aureus persistently colonizes the nasopharynx of about one-third of the human population, a key risk factor for community- and hospital-acquired invasive infections. Current strategies for $S$. aureus decolonization include topical and systemic administration of antibiotics, which is associated with selection for antibiotic resistance and posttreatment recolonization. Using a mouse model for $S$. aureus colonization, we show here that systemic administration of a recombinant monoclonal antibody neutralizing staphylococcal protein A (SpA) can stimulate antibacterial immunoglobulin $\mathrm{G}$ and immunoglobulin $\mathrm{A}$ responses and promote $S$. aureus decolonization. These results suggest that antibody neutralizing SpA, a B-cell superantigen, may also be useful for $S$. aureus decolonization in humans.

Keywords. Staphylococcus aureus; mouse model; monoclonal antibody; staphylococcal protein A; decolonization.

Staphylococcus aureus and its antibiotic-resistant isolates, designated MRSA (methicillin-resistant S. aureus), are a frequent cause of infectious diseases both in community and hospital settings [1]. The nasopharynx, gastrointestinal (GI) tract, and/or skin of about one-third of the human population are persistently colonized with $S$. aureus, and colonization is a key risk factor for community- and hospital-acquired invasive diseases [2]. Although asymptomatic colonization is common, $>80 \%$ of invasive $S$. aureus isolates are genetically indistinguishable from colonizing strains [2].

Current decolonization strategies aimed at reducing the risk of $S$. aureus infection, particularly for hospitalized individuals, include topical or systemic antibiotic treatment as well as

Received 22 July 2018; editorial decision 24 September 2018; accepted 19 October 2018; published online December 15, 2018.

Correspondence: 0 . Schneewind, MD, PhD, Department of Microbiology, University of Chicago, 920 E 58th St, Chicago, IL 60637 (oschnee@bsd.uchicago.edu).

The Journal of Infectious Diseases ${ }^{\circledR} \quad$ 2019;219:884-8

(C) The Author(s) 2018. Published by Oxford University Press for the Infectious Diseases Society of America. All rights reserved. For permissions, e-mail: journals.permissions@oup.com. DOI: 10.1093/infdis/jiy597 chlorine bleach baths [3]. Although such treatments have documented short-term success, they are associated with selection for antibiotic-resistant strains and recolonization. A US Food and Drug Administration-licensed vaccine or immune therapeutic that can prevent $S$. aureus colonization or invasive disease is currently not available. Recently, we developed $\mathrm{SpA}_{\mathrm{KKAA}}$, a nontoxigenic form of staphylococcal protein A (SpA), which elicits SpA-neutralizing antibodies [4]. These antibodies block the immunoglobulin G (IgG) Fc $\gamma$ binding attributes of SpA, promote opsonophagocytic killing of $S$. aureus, and provide protection in mouse models of staphylococcal bloodstream infection [4]. SpA-neutralizing antibodies also block the B-cell superantigen activity of SpA (cross-linking of immunoglobulin variable heavy 3 idiotype $\left[\mathrm{V}_{\mathrm{H}} 3\right]$ chains), thereby promoting broad-spectrum antibody (IgG and immunoglobulin A [IgA]) responses against many different staphylococcal antigens and providing immunity to recurrent disease in animals [5]. Staphylococcus aureus ST88 isolates persistently colonize the nasopharynx and GI tract of mice [6]. Expression of spa is a prerequisite for colonization and $\mathrm{SpA}_{\mathrm{KKAA}}$ immunization can decolonize mice, suggesting that the immune evasive attributes of $\mathrm{SpA}$ are also a prerequisite for staphylococcal commensalism [7]. Here we ask whether SpA-neutralizing monoclonal antibody (MAb) can be used for the decolonization of mice.

\section{MATERIALS AND METHODS}

\section{Recombinant mouse monoclonal antibody $3 \mathrm{~F} 6$}

Coding sequences of heavy and light chain genes of mouse hybridoma MAb (hMAb) 3F6-IgG2a [8] were synthesized in vitro and polymerase incomplete primer extension was used to swap genes into the expression vector pVITRO1-102.1F10-IgG1/ $\lambda$ (Addgene, number 50366). The recombinant plasmid was sequenced and transfected into FreeStyle 293-F cells (Life Technologies) using polyethylenimine. Transfectants were isolated with hygromycin B $(400 \mu \mathrm{g} / \mathrm{mL})$ selection and expanded in TripleFlask treated cultures. Cultures were centrifuged and conditioned supernatants subjected to affinity chromatography on protein A-sepharose to purify recombinant mouse MAb (rMAb) 3F6 [8].

\section{Enzyme-Linked Immunosorbent Assay}

Microtiter plates (Nunc Maxisorp) were coated with purified $\mathrm{SpA}_{\mathrm{KKAA}}$ or other antigens $(1 \mu \mathrm{g} / \mathrm{mL}$ in $0.1 \mathrm{M}$ carbonate buffer, $\mathrm{pH} 9.5$ at $4^{\circ} \mathrm{C}$ overnight). Wells were blocked and incubated with serum or antibodies and secondary antibody conjugates. To measure inhibition of SpA binding to human IgG (BioXCell), enzyme-linked immunosorbent assay (ELISA) plates were coated with $10 \mu \mathrm{g} \mathrm{SpA} / \mathrm{mL}$ overnight. After blocking, plates were incubated with $200 \mu \mathrm{g} / \mathrm{mL}$ of isotype control antibody (IgG2a, Fisher Scientific) or rMAb 3F6 or hMAb 3F6 prior to incubation 
with horseradish peroxidase (HRP)-conjugated human IgG ( $1 \mu \mathrm{g} / \mathrm{mL}$, Jackson ImmunoResearch). To measure binding to mouse neonatal $\mathrm{Fc}$ receptor $(\mathrm{mFcRn})$, ELISA plates were coated with streptavidin $(4 \mu \mathrm{g} / \mathrm{mL}$, New England Biolabs). After blocking, wells were incubated with biotinylated $\mathrm{mFcRn}(2 \mu \mathrm{g} / \mathrm{mL}$, Immunitrack) for 2 hours and with test antibodies at $\mathrm{pH} 6.0$ for 2 hours. All plates were incubated with HRP-conjugated secondary antibody specific to mouse IgG2a (Fisher Scientific) and developed using OptEIA reagent (BD Biosciences). Experiments were performed in triplicate to calculate averages and standard error of the mean, and repeated for reproducibility.

\section{Staphylococcal Survival in Blood}

MRSA strain MW2 and mouse-adapted S. aureus ST88 isolate WU1 were grown in tryptic soy broth, tryptic soy agar, or mannitol soy agar at $37^{\circ} \mathrm{C}$. To measure staphylococcal survival in vitro, isotype control (IgG2a), rMAb 3F6, or hMAb 3F6 was mixed with $50 \mu \mathrm{L}$ staphylococcal suspension, either $2.5 \times 10^{5}$ or $5 \times 10^{6}$ colony-forming units (CFU), to $0.5 \mathrm{~mL}$ of freshly drawn mouse or human blood, respectively. Blood was anticoagulated with $5 \mu \mathrm{g} / \mathrm{mL}$ desirudin and, where indicated, preincubated for 10 minutes with cytochalasin D. After incubation at $37^{\circ} \mathrm{C}$ for 0 minutes (mouse and human), 30 minutes (mouse), or 60 minutes (human), $0.5 \mathrm{~mL}$ phosphate-buffered saline containing $0.5 \%$ saponin, $100 \mathrm{U}$ streptokinase (SK), $50 \mu \mathrm{g}$ trypsin, $1 \mu \mathrm{g}$ DNase, and $5 \mu \mathrm{g}$ RNase (termed SK lysis buffer) were added to each sample for 10 minutes at $37^{\circ} \mathrm{C}$ prior to plating on agar for $\mathrm{CFU}$ enumeration. Assays were performed in duplicate and repeated for reproducibility.

\section{Animal Experiments}

rMAb 3F6, hMAb 3F6, or IgG2a control (5 mg/kg animal weight) was injected into the peritoneum of $\mathrm{BALB} / \mathrm{c}$ mice (groups of 10; 6-week-old female animals, Jackson Laboratory). After 16 hours, mice were anesthetized (ketamine-xylazine 100 $\mathrm{mg}-20 \mathrm{mg} / \mathrm{kg}$ ) and infected by injection into the periorbital venous plexus with $6.5 \times 10^{6} \mathrm{CFU}$ S. aureus USA400 (MW2). On day 15 following challenge, mice were killed by carbon dioxide inhalation and were necropsied, and staphylococcal load in homogenized renal tissue was quantified as CFU/g of tissue.

To measure antibody pharmacokinetics and dynamics, mice (groups of 5; 6-week-old C57BL/6J animals, Jackson Laboratory) were injected into the peritoneal cavity with antibody ( $5 \mathrm{mg} / \mathrm{kg}$ of body weight). After 1 and 6 hours as well as 1 , $2,3,7,11,15,22$, and 28 days, venous blood was retrieved periorbitally and plasma samples were analyzed by ELISA. Plasma antibody concentration was calculated via a standard curve of rMAb 3F6 diluted into mouse plasma at a range of 1-500 ng/ $\mathrm{mL}$. Antibody half-life was calculated using $\mathrm{N}(\mathrm{t})=\mathrm{N}_{0}(1 / 2)^{\mathrm{t} / \mathrm{t} / 2}$, where $\mathrm{N}_{0}$ is the highest concentration of $\mathrm{rMAb} 3 \mathrm{~F} 6, \mathrm{~N}(\mathrm{t})$ is the nondecayed concentration at time $t$, and $t_{1 / 2}$ is the half-life of the decaying concentration.
Mice (groups of 10, 6-week-old C57BL/6J animals) were administered $5 \mathrm{mg}$ antibody/ $\mathrm{kg}$ via intraperitoneal injection on days $0,14,28$, and 49 . Six hours after the first antibody injection, mice were inoculated intranasally with $1 \times 10^{8} \mathrm{CFU}$ of $S$. aureus strain WU1 [7]. On day 7 following inoculation (and in weekly intervals thereafter), the oropharynx of mice was swabbed, swabs were spread on mannitol soy agar, and plates were incubated for 16 hours at $37^{\circ} \mathrm{C}$ for CFU enumeration. On day 69, mice were bled and serum samples analyzed for IgG responses against the matrix of purified staphylococcal antigens [7]. In brief, $2 \mu \mathrm{g}$ purified antigen was spotted onto nitrocellulose, and membranes were blocked with 5\% degranulated milk and incubated with diluted mouse sera (1:1000 dilution) and IRDye 680-conjugated goat antimouse IgG (Li-cor, 1:10 000 dilution). Signal intensities were quantified using the Odyssey infrared imaging system (Li-cor) [4]. Li-cor technology was also used to quantify fecal IgA.

\section{Statistical Analysis}

Staphylococcal survival in blood and the inhibitory effects of antibodies were analyzed with the 2-tailed Student $t$ test. Bacterial loads in renal tissues were analyzed with the 2-tailed Mann-Whitney test. Two-way analysis of variance with Sidak multiple-comparison tests (GraphPad Software) was performed to analyze the statistical significance of mouse colonization and the staphylococcal antigen matrix data.

\section{RESULTS}

Earlier work isolated mouse hybridoma monoclonal 3F6 (hMAb 3F6), an IgG2a antibody that binds to the folded triple-helical structure of each of the 5 immunoglobulin-binding domains of $\mathrm{SpA}$, neutralizes their $\operatorname{IgG} \mathrm{Fc} \gamma$ and $\mathrm{V}_{\mathrm{H}} 3$ variant heavy chain binding activities and, when administered to mice, provides protection against $S$. aureus bloodstream infection [8]. As hybridomas are inherently unstable, we generated recombinant mouse rMAb 3F6 via plasmid transfection and expression in HEK293 F cells and affinity purification. When analyzed by Coomassiestained sodium dodecyl sulfate-polyacrylamide gel electrophoresis under reducing and nonreducing conditions, hMAb 3F6 and $\mathrm{rMAb} 3 \mathrm{~F} 6$ purified with similar abundance and integrity (Supplementary Figure 1). The hMAb 3F6 and rMAb 3F6 bound $\mathrm{SpA}_{\mathrm{KKAA}}$ with similar affinity, and both antibodies neutralized SpA binding to human IgG (Figure $1 \mathrm{~A}$ and $1 \mathrm{~B}$ ). Compared to mouse IgG2a control, addition of hMAb 3F6 or rMAb 3F6 to anticoagulated mouse or human blood reduced MRSA survival (Figure 1C). Antibody-mediated reduction of MRSA was abolished in blood samples treated with cytochalasin $\mathrm{D}$, an inhibitor of phagocytosis, indicating that hMAb 3F6 and rMAb 3F6 trigger opsonophagocytic killing of staphylococci. Administration of hMAb 3F6 and rMAb 3F6, but not IgG2a, to mice reduced the bacterial burden in kidney tissues (a key disease outcome measure), 15 days following bloodstream inoculation with 

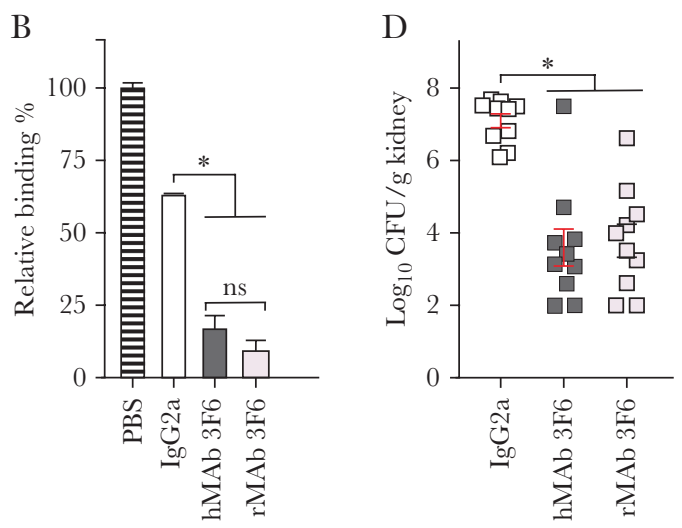

Figure 1. Recombinant mouse immunoglobulin G2a (IgG2a) monoclonal antibody rMAb 3F6 neutralizes staphylococcal protein A (SpA), promotes opsonophagocytic killing of Staphylococcus aureus, and protects mice against $S$. aureus bloodstream infection. $A$, Dissociation constants $\left(K_{\mathrm{d}}\right)$ were derived for rMAb $3 \mathrm{F6}$ and mouse hybridoma monoclonal antibody (hMAb) $3 \mathrm{~F} 6$ binding to $\mathrm{SpA}_{\mathrm{KKAA}}$. Antibodies $\left(0.15 \mathrm{mg} / \mathrm{mL}\right.$ ) were serially diluted across enzyme-linked immunosorbent assay plates coated with $\mathrm{SpA} \mathrm{KKAA}_{\mathrm{K}}$ $(1 \mu \mathrm{g} / \mathrm{mL})$ to calculate the dissociation constants using GraphPad Prism software. B, rMAb 3F6 and hMAb 3F6 prevent SpA binding to human IgG. Values were normalized to SpA interaction with human IgG in the absence of antibody $(\mathrm{n}=3)$. C, rMAb 3F6 and hMAb 3F6 promote opsonophagocytic killing of methicillin-resistant $S$. aureus (MRSA) isolate MW2 in mouse and human blood $(\mathrm{n}=3)$. $D$, Intraperitoneal administration of rMAb 3F6 and hMAb 3F6 antibodies (5 mg/kg body weight) into mice reduces replication of intravenously injected MRSA in the renal tissues of BALB/c mice. Error bars \pm standard error of the means were calculated from multiple independent repetitions of the experiments. ${ }^{*} P<.05$. Abbreviations: $A_{450}$, absorbance at $450 \mathrm{~nm}$; CD, cytochalasin D; CFU, colony-forming units; hMAb, mouse hybridoma monoclonal antibody; IgG2a, immunoglobulin G2a; ns, not significant; PBS, phosphate-buffered saline; rMAb, mouse recombinant monoclonal antibody; SpA, staphylococcal protein A.

MRSA (Figure 1D). Together these data indicate that rMAb 3F6 exhibits similar antigen affinity, target neutralizing, and opsonophagocytic killing activity as well as disease prevention as hMAb 3F6.

In vivo pharmacodynamics of antibodies are critically dependent on the FcRn, a heterodimer with a major histocompatibility complex class I-like $\alpha$-chain and $\beta-2$ microglobulin, which captures IgG phagocytosed into endosomes or within renal filtrates under acidic conditions ( $\mathrm{pH}$ 6.0) for subsequent release at $\mathrm{pH}$ neutral conditions [9]. At $\mathrm{pH}$ 6.0, the dissociation constant $\left(\mathrm{K}_{d}\right)$ of hMAb 3F6 and rMAb 3F6 for mouse FcRn was similar; however, rMAb 3F6 exhibited higher affinity for the receptor than hMAb 3F6 (Figure 2A). Intraperitoneal injection of $\mathrm{rMAb}$ 3F6 into mice was associated with a biexponential plasma concentration-time profile including a short distribution phase followed by a long elimination phase (Figure 2B). Plasma concentration of rMAb $3 \mathrm{~F} 6$ peaked 6 hours postinjection and after 28 days was $58 \mathrm{nM}$ with a calculated half-life of 8.6 days (Figure 2B).
Intranasal inoculation of C57BL/6J mice with S. aureus WU1 leads to persistent colonization of the nasopharynx and GI tract, which can be monitored by enumerating staphylococci in pharyngeal swab and fecal samples in weekly intervals [7]. Staphylococcus aureus spa colonizes mice similar to wild-type staphylococci; however, spa mutants elicit pathogen-specific IgG responses against key colonization determinants that are associated with decolonization of staphylococci from the nasopharynx and GI tract within 14-28 days [7]. Intraperitoneal administration of rMAb 3F6 prior to $S$. aureus inoculation triggered decolonization of staphylococci from the pharynx beginning on day 21 (Figure 2C). Administration of control IgG2a did not affect S. aureus colonization. Analysis of mouse serum for S. aureus-specific IgG revealed that decolonized mice produced increased antibody titers against the colonization determinants ClfB, IsdA, and SasG as well as several other surface antigens of staphylococci (Figure 2D and Supplementary Table 1). Intraperitoneal administration of rMAb 3F6 7 days after S. aureus colonization also produced increased IgG titers against staphylococcal antigens and prompted decolonization of staphylococci 
A

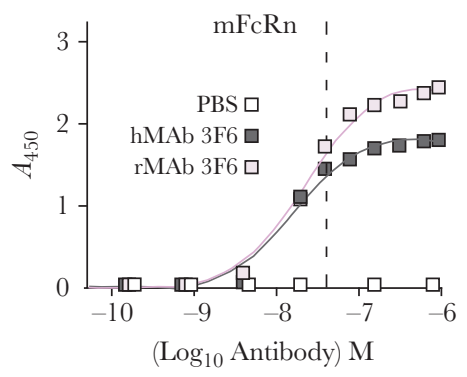

B

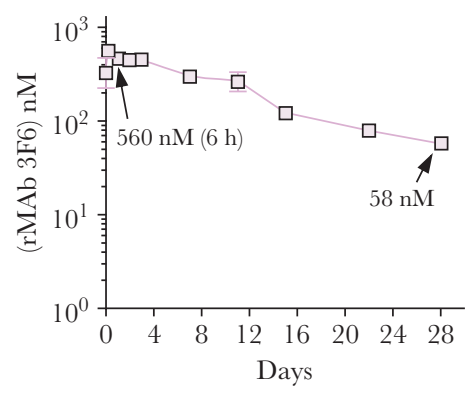

C

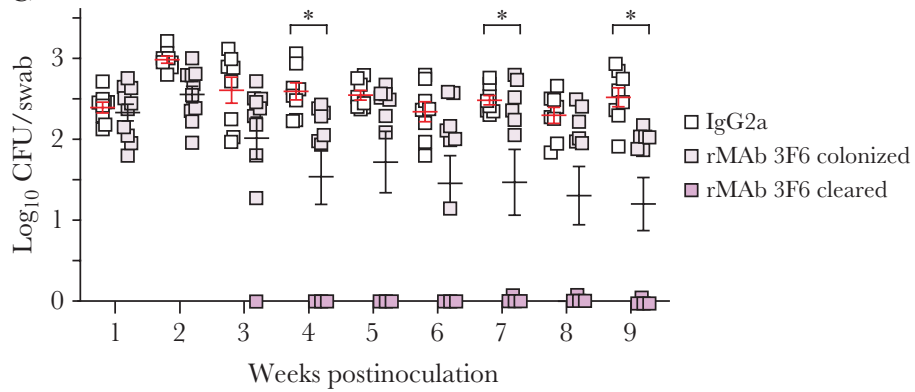

$\mathrm{D}$

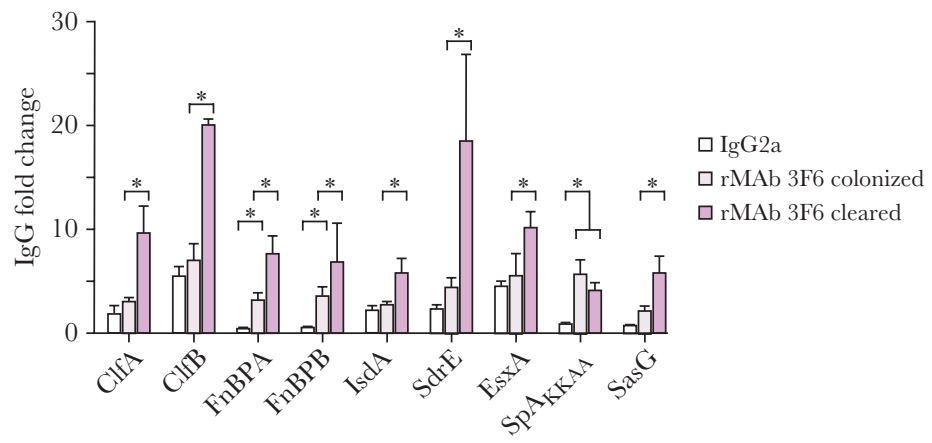

Figure 2. Administration of mouse recombinant monoclonal antibody (rMAb) 3F6 promotes Staphylococcus aureus decolonization in mice. A, Enzyme-linked immunosorbent assay examining rMAb 3F6 and mouse hybridoma monoclonal antibody (hMAb) 3F6 affinity for the mouse neonatal Fc receptor. Dissociation constants $\left(K_{d}\right)$ were calculated using GraphPad Prism software. B, Plasma concentration-time profile of rMAb $3 F 6$ following intraperitoneal administration into C57BL/6 mice ( $\mathrm{n}=5)$. C Intraperitoneal administration of rMAb $3 F 6$ prior to nasal inoculation with $S$. aureus WU1 promotes progressive decolonization of staphylococci from the nasopharynx of C57BL/6 mice $(n=10)$. The median and standard deviation for each group of animals on a given day are indicated by the horizontal lines and error bars. $D$, Administration of rMAb 3F6 prior to colonization promotes enhanced antistaphylococcal serum immunoglobulin $\mathrm{G}$ responses. Sera of animals shown in $(C)$ were tested for antibodies against the indicated $S$. aureus antigens. Error bars \pm standard error of the mean were calculated from multiple independent repetitions of the experiments. ${ }^{*} P<.05$. Abbreviations: $A_{450}$, absorbance at $450 \mathrm{~nm}$; CFU, colony-forming units; hMAb, mouse hybridoma monoclonal antibody; IgG, immunoglobulin G; IgG2a, immunoglobulin G2a; mFcRn, mouse neonatal Fc receptor; PBS, phosphate-buffered saline; rMAb, mouse recombinant monoclonal antibody; SpA, staphylococcal protein A.

from the pharynx and GI tract beginning 14 days after treatment (Supplementary Figure 2 and Supplementary Table 2). When analyzed for intestinal IgA, rMAb 3F6 treatment and decolonization were associated with increased IgA against staphylococcal surface antigens (Supplementary Table 3).

\section{DISCUSSION}

Nasopharyngeal colonization with Haemophilus influenza, Neisseria meningitidis, Streptococcus pneumoniae, and S. aureus is a risk factor for invasive human diseases [10]. Naturally acquired antibodies, that is, plasma IgG specific for capsular polysaccharide of $H$. influenzae, N. meningitidis, and S. pneumoniae, promote decolonization and also protect against invasive diseases caused by these microbes [10]. Staphylococcus aureus strains elaborate 2 serologically distinct capsular polysaccharides, which are, however, not essential for either nasopharyngeal colonization or invasive staphylococcal disease [11, 12]. Further, immunization of humans with staphylococcal capsular conjugate elicits specific plasma IgG, but these antibodies do not protect against invasive disease or colonization $[13,14]$. Earlier work demonstrated that $\mathrm{SpA}$, a B-cell superantigen expressed on the surface of all colonizing $S$. aureus isolates, is required for nasopharyngeal colonization of humans and mice $[7,15]$. SpA promotes B-cell proliferation and the secretion of $\mathrm{V}_{\mathrm{H}} 3$ clonal antibodies of different subclasses that fail to recognize $S$. aureus antigens $[5,10]$. Here we show that SpA-neutralizing MAb can promote pathogen-specific antibody responses that result in decolonization of S. aureus from the nasopharynx and GI tract of mice. We hypothesize that decolonization is based on MAb removal of circulating SpA, which is persistently released by colonizing bacteria, thereby promoting $\operatorname{IgG}$ and $\operatorname{IgA}$ responses against key $S$. aureus surface determinants that otherwise bind specific host factors and contribute to colonization. Unlike humans, where $>50 \%$ of $\mathrm{B}$ cells present $\mathrm{V}_{\mathrm{H}} 3$ clonal B-cell receptors (BCRs), $<10 \%$ of mouse $B$ cells present $V_{H} 3 B C R s$ responsive to $\mathrm{SpA}$ [10]. Assuming B cells of humans colonized with S. aureus respond to the immune stimulatory attributes of SpA-neutralizing antibody, humanized MAb 3F6 may be useful for both S. aureus decolonization and the prevention of invasive disease, particularly for high-risk individuals in hospital settings.

\section{Supplementary Data}

Supplementary materials are available at The Journal of Infectious Diseases online. Consisting of data provided by 
the authors to benefit the reader, the posted materials are not copyedited and are the sole responsibility of the authors, so questions or comments should be addressed to the corresponding author.

\section{Notes}

Financial support. This work was supported by the National Institute of Allergy and Infectious Diseases (grant numbers AI038897 and AI052747 to O. S.); the Shenzhen Science and Technology Innovation Commission Project (grant number JCYJ20160229153541081 to Shenzhen International Institute for Biomedical Research); and project grant number CYZZ20160226111115783 to Immunartis LLC.

Potential conflicts of interest. The authors declare conflicts of interests as inventors of patents under commercial license for development of antibody therapeutics against $S$. aureus infections. All authors have submitted the ICMJE Form for Disclosure of Potential Conflicts of Interest. Conflicts that the editors consider relevant to the content of the manuscript have been disclosed.

\section{References}

1. Tong SY, Davis JS, Eichenberger E, Holland TL, Fowler VG Jr. Staphylococcus aureus infections: epidemiology, pathophysiology, clinical manifestations, and management. Clin Microbiol Rev 2015; 28:603-61.

2. van Belkum A, Melles DC, Nouwen J, et al. Co-evolutionary aspects of human colonisation and infection by Staphylococcus aureus. Infect Genet Evol 2009; 9:32-47.

3. Liu C, Bayer A, Cosgrove SE, et al. Clinical practice guidelines by the Infectious Diseases Society of America for the treatment of methicillin-resistant Staphylococcus aureus infections in adults and children: executive summary. Clin Infect Dis 2011; 52:285-92.

4. Kim HK, Cheng AG, Kim HY, Missiakas DM, Schneewind O. Nontoxigenic protein A vaccine for methicillin-resistant Staphylococcus aureus infections in mice. J Exp Med 2010; 207:1863-70.
5. Pauli NT, Kim HK, Falugi F, et al. Staphylococcus aureus infection induces protein A-mediated immune evasion in humans. J Exp Med 2014; 211:2331-9.

6. Schulz D, Grumann D, Trübe P, et al. Laboratory mice are frequently colonized with Staphylococcus aureus and mount a systemic immune response-note of caution for in vivo infection experiments. Front Cell Infect Microbiol 2017; 7:152.

7. Sun Y, Emolo CE, Holtfreter S, et al. Staphylococcal protein $\mathrm{A}$ is required for persistent colonization of mice with Staphylococcus aureus. J Bacteriol 2018; 200:e00735-17.

8. Kim HK, Emolo C, DeDent AC, Falugi F, Missiakas DM, Schneewind O. Protein A-specific monoclonal antibodies and prevention of Staphylococcus aureus disease in mice. Infect Immun 2012; 80:3460-70.

9. Raghavan M, Bjorkman PJ. Fc receptors and their interactions with immunoglobulins. Annu Rev Cell Dev Biol 1996; 12:181-220.

10. Missiakas D, Schneewind O. Staphylococcus aureus vaccines: deviating from the carol. J Exp Med 2016; 213:1645-53.

11. Cheng AG, Kim HK, Burts ML, Krausz T, Schneewind O, Missiakas DM. Genetic requirements for Staphylococcus aureus abscess formation and persistence in host tissues. FASEB J 2009; 23:3393-404.

12. Misawa Y, Kelley KA, Wang X, et al. Staphylococcus aureus colonization of the mouse gastrointestinal tract is modulated by wall teichoic acid, capsule, and surface proteins. PLoS Pathog 2015; 11:e1005061.

13. Shinefield H, Black S, Fattom A, et al. Use of a Staphylococcus aureus conjugate vaccine in patients receiving hemodialysis. N Engl J Med 2002; 346:491-6.

14. Creech CB 2nd, Johnson BG, Alsentzer AR, Hohenboken M, Edwards KM, Talbot TR 3rd. Vaccination as infection control: a pilot study to determine the impact of Staphylococcus aureus vaccination on nasal carriage. Vaccine 2009; 28:256-60.

15. Cole AL, Muthukrishnan G, Chong C, et al. Host innate inflammatory factors and staphylococcal protein A influence the duration of human Staphylococcus aureus nasal carriage. Mucosal Immunol 2016; 9:1537-48. 